\title{
Paget Disease of the Anus
}

National Cancer Institute

\section{Source}

National Cancer Institute. Paget Disease of the Anus. NCI Thesaurus. Code C5598.

A slowly spreading, erythematous eczematoid plaque in the anal region. Histologically, the basal part or whole thickness of the squamous epithelium is infiltrated by large cells with abundant pale cytoplasm and large nuclei. Half of the cases are associated with an internal malignancy, most often a colorectal adenocarcinoma. The other half of the cases, have a high local recurrence rate and they may become invasive (WHO). 